\title{
Trans-Planckian Physics and Non-Commutative Inflation
}

\author{
Seoktae Koh \\ Department of Physics, Hanyang University, \\ Seoul 133-791, Korea \\ and Institute of Theoretical Physics, \\ Chinese Academy of Sciences, \\ P.O. Box 2735, Beijing, 100080, China \\ skoh@itp.ac.cn
}

\begin{abstract}
Non-commutativity of spacetime at the Planck scale may deform the usual dispersion relations. And these deformed dispersion relations could lead to the accelerating phase without a scalar field. In this paper, we have calculated the spectral index and the running of spectral index in a non-commutative inflation model. Non-commutative inflation with thermal radiation gives a scale invariant spectrum in the limit $w \rightarrow-1$ and negative running spectral index which are consistent with the WMAP 3-year results.
\end{abstract}




\section{INTRODUCTION}

It is realized from the fundamental theory (e.g. string theory) that spacetime structure will become non-commutative at the Planck scale. And it is also believed that inflation can provide a promising framework to probe Planck scale physics through the prediction of cosmological perturbations which arise due to the non-commutativity. There are several attempts to show the effect of the non-commutativity of the spacetime on the cosmological observations [1] [2] [3].

Spacetime non-commutativity may result in deformed dispersion relations [4]. It was

pointed out in Ref. [1] that these deformed dispersion relations lead to a period of inflation driven by non-commutative radiation instead of usual scalar fields. Its distinct features are thermal fluctuations as seeds for structure formation and the existence of two branches in the deformed dispersion relation. High energy branch ,in which the energy decrease as the momentum increases, leads to an accelerated expansion phase. The resulting spectrum of the cosmological perturbations in this model produced a scale invariant spectrum in the limit $w \rightarrow-1[5]$.

The following section provides a brief review of the non-commutative inflation model and section III gives a detail calculation of the power spectrum in this model. In section IV], we calculate a spectral index and a running of spectral index and then compare with the observational data. And finally we conclude with a brief discussion.

\section{BRIEF REVIEW OF NON-COMMUTATIVE INFLATION}

The modified dispersion relations for massless particles which result from the space-time non-commutativity,

$$
E^{2}-p^{2} c^{2} f(E)^{2}=0
$$

drive the accelerated expansion of space. Here,

$$
f(E)=1+(\lambda E)^{\alpha}
$$

where $p$ and $E$ denote momentum and energy, respectively, $\alpha \geq 1$ is a positive constant, and $\lambda$, which determines the maximal momentum, has a length scale. 


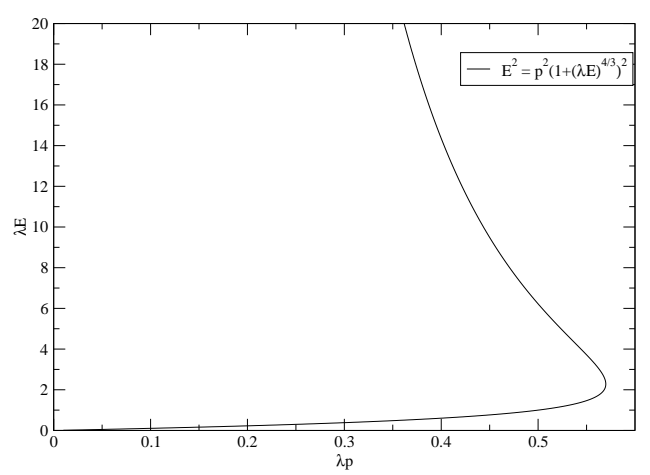

FIG. 1: Modified dispersion relations due to the non-commutativity of spacetime for $\alpha=4 / 3$. While for a low energy branch energy increases as momentum increases, as momentum increases, energy decrease in a high energy branch.

These dispersion relations have two branches for $\alpha>1$ (see Fig. 1). For the high energy branch, the energy increases as the momentum decreases. This behavior can make it possible to generate accelerated expansion phase.

The energy density with the modification of the dispersion relations is given by

$$
\rho=\frac{1}{\pi^{2}} \int d E \frac{E^{3}}{e^{E / T}-1} \frac{1}{f^{3}}\left|1-\frac{f^{\prime} E}{f}\right|,
$$

and the pressure is

$$
\mathcal{P}=\frac{1}{3 \pi^{2}} \int \frac{d E}{1-\frac{f^{\prime} E}{f}} \frac{E^{3}}{e^{E / T}-1} \frac{1}{f^{3}}\left|1-\frac{f^{\prime} E}{f}\right|,
$$

where $f^{\prime} \equiv d f / d E$. While for low temperatures $\rho \propto T^{4}$ as usual radiation does, $\rho \propto T$ for $\lambda T \gg 1[4]$. In the high energy limit $(\lambda E \gg 1), f^{\prime} E / f \simeq \alpha$ and the equation of state parameter $w$ takes on negative values,

$$
w \simeq \frac{1}{3(1-\alpha)}
$$

As temperature drops, the equation of state of non-commutative radiation changes from that of an accelerated universe to $\frac{1}{3}$ which is an equation of state of ordinary radiation. Thus, there is no need for any period of reheating.

The Friedmann and energy conservation equations for the non-commutative radiation are

$$
\begin{aligned}
\left(\frac{\dot{a}}{a}\right)^{2} & =\frac{8 \pi}{3 M_{p}} \rho, \\
\dot{\rho} & =-3 \frac{\dot{a}}{a}(1+w) \rho .
\end{aligned}
$$


For a constant equation of state, the background solutions can be written as

$$
\rho \propto a^{-3(1+w)}, \quad a \propto t^{2 / 3(1+w)} \propto \eta^{2 /(1+3 w)},
$$

where $\eta$ is a conformal time $(d t=a d \eta)$. Inflation ends when there are no more excited states on the high energy branch of the modified dispersion relations.

\section{DENSITY PERTURBATIONS IN NON-COMMUTATIVE INFLATION}

We work in the longitudinal gauge to calculate the density perturbations of noncommutative fluid. In this gauge, the metric takes the form

$$
d s^{2}=a^{2}\left[-(1+2 \Phi)+(1-2 \Psi) \gamma_{i j} d x^{i} d x^{j}\right]
$$

where the functions $\Phi$ (the Bardeen potential[6]) and $\Psi$ describe the scalar metric fluctuations.

Using the variable $v$ which is defined as[7]

$$
v=\frac{a}{c_{s} \sqrt{\rho+\mathcal{P}}}\left(\delta q-\frac{\rho+\mathcal{P}}{H} \Phi\right)=-z \zeta, \quad z=\frac{a \sqrt{\rho+\mathcal{P}}}{H c_{s}}
$$

the equation of motion for scalar metric perturbations takes the simple form

$$
v_{k}^{\prime \prime}+\left(k^{2} c_{s}^{2}-\frac{z^{\prime \prime}}{z}\right) v_{k}=0,
$$

where $c_{s}^{2} \equiv \delta \mathcal{P} / \delta \rho$ is a sound speed, $\delta q$ a velocity perturbation and a prime denotes a derivative with respect to conformal time $\eta$. The curvature perturbation $\zeta$ on uniform energy density hypersurface, which is conserved on scales larger than the sound horizon for purely adiabatic perturbations, is related to $\Phi$ as

$$
\zeta=\Phi-\frac{H}{\dot{H}}(\dot{\Phi}+H \Phi) .
$$

Then the power spectrum of $\zeta$ is given by

$$
\mathcal{P}_{\zeta}(k)=\frac{k^{3}}{2 \pi^{2}}\left\langle\theta\left|\zeta_{k}^{*} \zeta_{k}\right| \theta\right\rangle=\frac{k^{3}}{2 \pi^{2}} \frac{\left\langle\theta\left|v_{k}^{*} v_{k}\right| \theta\right\rangle}{z^{2}}
$$

The seeds for large scale structures in this non-commutative inflation model are thermal fluctuations instead of quantum fluctuations which are responsible for structure formation in the conventional scalar driven inflation models. We need initial conditions to calculate 
completely $v_{k}$ or curvature perturbations $\zeta_{k}$ at superhorizon scales. For a fixed scale $k$, we choose thermal correlation length to impose initial conditions [5]

$$
T^{-1}\left(\eta_{i}(k)\right)=a\left(\eta_{i}(k)\right) / k
$$

since on scale larger than that the interactions are not likely able to maintain thermal equilibrium. Thermal state $|\theta\rangle$ at time $\eta_{i}(k)$ is determined by $n_{k}=\left\langle\theta\left|a_{k}^{\dagger} a_{k}\right| \theta\right\rangle=1 /\left(e^{E / a T}-\right.$ 1).

In the Heisenberg picture, the Hamiltonian $H_{k}$ is given by

$$
H_{k}=\int d^{3} k \frac{1}{2}\left[k\left(a_{k} a_{k}^{\dagger}+a_{k}^{\dagger} a_{k}\right)+i \frac{z_{k}^{\prime}}{z_{k}}\left(a_{k}^{\dagger} a_{-k}^{\dagger}-a_{k} a_{-k}\right)\right] .
$$

The annihilation $a_{k}$ and creation operator $a_{k}^{\dagger}$ at time $\eta$ are related to those at the initial time $\eta_{i}$ by the Bogoliubov transformation

$$
\begin{aligned}
a_{k}(\eta) & =\alpha_{k}(\eta) a_{k}\left(\eta_{i}\right)+\beta_{k}(\eta) a_{-k}^{\dagger}\left(\eta_{i}\right), \\
a_{-k}^{\dagger}(\eta) & =\alpha_{k}^{*}(\eta) a_{-k}^{\dagger}\left(\eta_{i}\right)+\beta_{k}^{*}(\eta) a_{k}\left(\eta_{i}\right),
\end{aligned}
$$

where $\alpha_{k}$ and $\beta_{k}$ are the Bogoliubov coefficients which satisfy the normalization condition $\left|\alpha_{k}\right|^{2}-\left|\beta_{k}\right|^{2}=1$. The canonical variable $v_{k}$ and its conjugate momenta $\pi_{k}$ yields

$$
\begin{aligned}
& v_{k}(\eta)=f_{k}(\eta) a_{k}\left(\eta_{i}\right)+f_{k}^{*} a_{-k}^{\dagger}\left(\eta_{i}\right), \\
& \pi_{k}(\eta)=-i\left[g_{k}(\eta) a_{k}\left(\eta_{i}\right)+g_{k}^{*}(\eta) a_{-k}^{\dagger}\left(\eta_{i}\right)\right],
\end{aligned}
$$

where

$$
\begin{aligned}
& f_{k}(\eta)=\frac{1}{\sqrt{2 k c_{s}}}\left(\alpha_{k}(\eta)+\beta_{k}^{*}(\eta)\right) \\
& g_{k}(\eta)=\sqrt{\frac{k c_{s}}{2}}\left(\alpha_{k}(\eta)-\beta_{k}^{*}(\eta)\right)
\end{aligned}
$$

For a power law inflation with a constant $w$, the mode equation has the following solution

$$
f_{k}(\eta)=A_{k} \sqrt{-\eta} J_{\nu}\left(-k c_{s} \eta\right)+B_{k} \sqrt{-\eta} Y_{\nu}\left(-k c_{s} \eta\right)
$$

where $J_{\nu}$ and $Y_{\nu}$ are Bessel functions of the first and second kind, respectively, and we have used

$$
\frac{z^{\prime \prime}}{z}=\frac{\nu^{2}-1 / 4}{\eta^{2}}, \quad \nu^{2}=\frac{1}{4}+\frac{2(1-3 w)}{(1+3 w)^{2}}
$$


The expression of $A_{k}$ and $B_{k}$ can be obtained from the normalization condition for $\alpha_{k}$ and $\beta_{k}$ and $\beta_{k}^{*}\left(\eta_{i}\right)=0$ at the initial time $\eta_{i}$

$$
\begin{aligned}
\left|A_{k}\right|^{2}=-\frac{\pi^{2}}{8} k c_{s} \eta_{i}\left(Y_{\nu}^{2}\left(-k c_{s} \eta_{i}\right)+Y_{\nu-}^{2}\left(-k c_{s} \eta_{i}\right)\right) \\
\left|B_{k}\right|^{2}=-\frac{\pi^{2}}{8} k c_{s} \eta_{i}\left(J_{\nu}^{2}\left(-k c_{s} \eta_{i}\right)+J_{\nu-1}^{2}\left(-k c_{s} \eta_{i}\right)\right) \\
A_{k} B_{k}^{*}=-i \frac{\pi}{4}+\frac{\pi^{2}}{8} k c_{s} \eta_{i}\left(J_{\nu}\left(-k c_{s} \eta_{i}\right) Y_{\nu}\left(-k c_{s} \eta_{i}\right)\right. \\
\left.\quad+J_{\nu-1}\left(-k c_{s} \eta_{i}\right) Y_{\nu-1}\left(-k c_{s} \eta_{i}\right)\right) .
\end{aligned}
$$

In the large scale limit, $k c_{s} \eta \ll 1$, Bessel functions have asymptotic form as

$$
J_{\nu}\left(-k c_{s} \eta\right) \simeq \frac{1}{\Gamma(\nu+1)}\left(-\frac{k c_{s} \eta}{2}\right)^{\nu}, \quad Y_{\nu}\left(-k c_{s} \eta\right) \simeq-\frac{\Gamma(\nu)}{\pi}\left(-\frac{k c_{s} \eta}{2}\right)^{-\nu},
$$

where $\nu=3(\omega-1) / 2(1+3 \omega)$ from (19)). Then curvature perturbation in the large scale limit can be written as

$$
\zeta_{k}(\eta) \simeq c_{k}+d_{k} \eta^{-\frac{3(1-w)}{1+3 w}}
$$

where $c_{k}$ and $d_{k}$ are growing and decaying mode coefficients, respectively. As expected, $\zeta_{k}$ has a constant solution in the large scale limit [8].

Using (16) and (18), the power spectrum (12) of $\zeta_{k}$ at the time of Hubble radius crossing can now be calculated as

$$
\begin{aligned}
\mathcal{P}_{\zeta}(k) & =\frac{k^{3}}{2 \pi^{2}} \frac{\left|f_{k}\right|^{2}}{z^{2}} \operatorname{coth}\left(\frac{k c_{s}}{a_{i} T_{i}}\right) \\
& \sim \frac{2 \pi}{3 M_{p}^{4}} \frac{k^{2} c_{s}^{2} \rho\left(\eta_{h}\right) \eta_{i} \eta_{h}}{\epsilon c_{s}} \\
& \sim \frac{2 \pi^{2}}{3 M_{p}^{4}} \frac{\rho\left(\eta_{h}\right)}{\epsilon} \frac{T_{i}}{c_{s} H_{i}},
\end{aligned}
$$

where we have used $z \simeq M_{p} a \epsilon^{1 / 2} / \sqrt{4 \pi c_{s}^{2}}$ and $\eta_{i} \simeq 1 /\left(a_{i} H_{i}\right)$ and $\eta_{h} \simeq 1 /\left(a_{i} T_{i}\right)$ since $k c_{s}=$ $a_{i} T_{i}=a\left(\eta_{h}\right) H\left(\eta_{h}\right)$. For later use, we have defined slow-roll parameters

$$
\epsilon \equiv-\frac{\dot{H}}{H^{2}}, \quad \eta \equiv \frac{\ddot{H}}{H \dot{H}}, \quad \xi \equiv \frac{H^{(3)}}{H^{2} \dot{H}},
$$

where $H^{(3)} \equiv d^{3} H / d t^{3}$. 


\section{OBSERVATIONS}

One important feature of the thermal non-commutative inflation model is the existence of two branches in dispersion relations. This can make it possible to generate accelerated expansion phase and, after a period of inflation, change into the usual radiation dominated phase smoothly. In order to differentiate between non-commutative inflation model and usual scalar field-driven inflation model, it is necessary to constrain the power spectrum of the cosmological perturbations

$$
\ln \mathcal{P}_{\zeta}(k)=\ln \mathcal{P}_{\zeta}\left(k_{0}\right)+\left(n_{s}-1\right) \ln \frac{k}{k_{0}}+\frac{1}{2} \alpha_{s} \ln ^{2} \frac{k}{k_{0}}+\cdots,
$$

where $n_{s}$ is a spectral index of the spectrum and $\alpha_{s}$ a running of the spectral index, respectively. Three-year WMAP results[9] show that

$$
n_{s}=1.16 \pm 0.10, \quad \alpha_{s}=-0.085 \pm 0.043
$$

on the scale $k_{0}=0.002 \mathrm{Mpc}^{-1}[15]$.

Here we briefly mention the observational implications of the thermal non-commutative inflation model. It is convenient to use the following relations to calculate the spectral index and its running:

$$
\frac{d \ln \rho}{d \ln k}=-2 \epsilon, \quad \frac{d \epsilon}{d \ln k}=2 \epsilon^{2}-\epsilon \eta, \quad \frac{d \eta}{d \ln k}=\xi+\epsilon \eta-\eta^{2},
$$

where we have used $d \ln k \simeq H d t$ when the modes cross the Hubble radius. Then the spectral index from (23) becomes

$$
n_{s}-1 \equiv \frac{d \ln \mathcal{P}_{\zeta}(k)}{d \ln k}=-4 \epsilon+\eta+\frac{d}{d \ln k} \ln \left(\frac{T_{i}}{c_{s} H_{i}}\right)
$$

For a constant equation of state $(\dot{w} \ll w)$, using Eq. (71) slow-roll parameters become

$$
\begin{aligned}
& \epsilon \simeq \frac{3}{2}(1+w), \quad \eta \simeq-3(1+w), \quad \xi \simeq \frac{27}{2}(1+w)^{2}, \\
& \frac{d}{d \ln k} \ln \left(\frac{T_{i}}{c_{s} H_{i}}\right) \sim \frac{3(1+w)}{2(2+3 w)},
\end{aligned}
$$

where we have used $\rho \propto T$ during inflation to derive Eq. (30). Then the spectral index (28) becomes

$$
n_{s}-1 \simeq-\frac{3(1+w)(18 w+11)}{2(2+3 w)}
$$


This gives a scale invariant spectrum in the limit $w \rightarrow-1$ with a slightly red tilt[5] [16]. It seems to be inconsistent with the WMAP three-year data which favor $n_{s}>1$ on the large scales.

Using the relations (27), the running of spectral index $\alpha_{s}$ becomes

$$
\begin{aligned}
\alpha_{s} & \equiv \frac{d n_{s}}{d \ln k}=-8 \epsilon^{2}+5 \epsilon \eta-\eta^{2}+\xi+\frac{d^{2}}{d \ln k^{2}} \ln \left(\frac{T_{i}}{c_{s} H_{i}}\right) \\
& \simeq-36(1+w)^{2},
\end{aligned}
$$

where we have used a constant equation of state to derive the second line in the above expression. This gives a negative value of running of spectral index which is consistent with the observational data.

Another important observational signature is a non-Gaussianity of cosmological perturbations[10]. Recent study[11] shows that non-Gaussianity at $95 \%$ confidence level is in the region

$$
26.91<f_{N L}<146.71
$$

where non-Gaussianity parameter $f_{N L}$ is defined as

$$
\zeta=\zeta_{g}+\frac{3}{5} f_{N L}\left(\zeta_{g}^{2}-\left\langle\zeta_{g}^{2}\right\rangle\right)
$$

Here, subscript $g$ denotes the Gaussian part of $\zeta$. Detection of non-Gaussianity from the future satellite experiment can discriminate between different inflation models.

Interestingly, in Ref. [12], it was shown that thermal fluctuations may lead to a large non-Gaussianity. The non-Gaussianity parameter takes the form in the thermal fluctuation model

$$
f_{N L}=\frac{5 \epsilon m}{72 \pi G m \rho L^{2}}
$$

where $\rho \propto T^{m}$ and $L$ is a thermal length scale. This result implies that as $L$ is smaller, $f_{N L}$ can be larger. The non-Gaussianity in thermal non-commutative inflation model is under considerations. [13]

\section{CONCLUSION AND DISCUSSIONS}

In this paper we have considered the inflation model which arises due to the noncommutativity of spacetime in the early universe and calculated the spectral index and 
the running of spectral index in this model. Non-commutativity of spacetime at the Planck scale may deform usual dispersion relations. Further, these modified dispersion relations make it possible to obtain a period of inflation driven by non-commutative radiation. While the seeds for large scale structure in the usual scalar field driven inflation model are quantum fluctuations, those are thermal fluctuations in non-commutative inflation. Keeping these in mind, it seems natural to impose the initial conditions at thermal correlation length to maintain thermal equilibrium. In Ref. [5], it was shown that this inflation model can give a scale invariant spectrum in the limit $w \rightarrow-1$.

One of the important features of this model is the existence of two branches. In the high energy limit, energy decreases as momentum increases. This property is crucial in realizing accelerated expansion phase in modified dispersion relations. On the contrary, as momentum decreases, energy decreases in the low energy limit as in usual dispersion relations. Thus, as temperature drops, the accelerated expansion phase change into the usual radiation dominated phase $(w=1 / 3)$ smoothly without need of reheating.

It might be worth to find the observational signal of these distinct features of noncommutative inflation model to discriminate from the usual scalar field driven inflation model. We thus have calculated the spectral index and the running of spectral index. With the slow-roll approximation, $n_{s}-1 \sim-9(1+w)$ and $\alpha_{s} \sim-36(1+w)^{2}$. These show that power spectrum is scale invariant as long as $\epsilon, \eta \ll 1$ (or $w \rightarrow-1$ ), which is a necessary condition for occurring enough inflation, and has negative running of spectral index. While the running of spectral index is consistent with data, the spectral index seems to be inconsistent since WMAP three-year data favor $n_{s}>1$.

Another important observational signal is a non-Gaussianity of cosmological perturbations whose detection will be an important mission in the future satellite experiment. Thermal fluctuations can lead to a large non-Gaussianity [12] [14] if thermal length scale is smaller than Horizon scale at the horizon crossing. The non-Gaussianity in this non-commutative inflation model is under considerations [13].

These observational signals can provide the possibility to probe Planck scale physics in the current observations by the induced signals in the spectrum of cosmological perturbations. 


\section{Acknowledgments}

The author would like to thank the organizers for inviting me and their warm hospitality during CosPA 2007 at National Taiwan University. And the author also thank R. Brandenberger and R. Cai for useful comments. This work was supported by the Korea Science and Engineering Foundation (KOSEF) (No. R01-2006-000-10651-0).

[1] S. Alexander, R. Brandenberger and J. Magueijo, Phys. Rev. D 67, 081301 (2003) arXiv:hep-th/0108190].

[2] R. Brandenberger and P. M. Ho, Phys. Rev. D 66, 023517 (2002) [AAPPS Bull. 12N1, 10 (2002)] arXiv:hep-th/0203119].

[3] H. C. Kim, J. H. Yee and C. Rim, Phys. Rev. D 72, 103523 (2005) arXiv:gr-qc/0506122].

[4] S. Alexander and J. Magueijo, arXiv:hep-th/0104093.

[5] S. Koh and R. H. Brandenberger, JCAP 0706, 021 (2007) arXiv:hep-th/0702217].

[6] J. M. Bardeen, Phys. Rev. D 22, 1882 (1980).

[7] V. F. Mukhanov, H. A. Feldman and R. H. Brandenberger, Phys. Rept. 215, 203 (1992).

[8] S. Koh and R. H. Brandenberger, JCAP 0711, 013 (2007) [arXiv:0708.1014 [hep-th]].

[9] D. N. Spergel et al. [WMAP Collaboration], Astrophys. J. Suppl. 170, 377 (2007) arXiv:astro-ph/0603449].

[10] J. M. Maldacena, JHEP 0305, 013 (2003) arXiv:astro-ph/0210603]; N. Bartolo, E. Komatsu, S. Matarrese and A. Riotto, Phys. Rept. 402, 103 (2004) [arXiv:astro-ph/0406398].

[11] A. P. S. Yadav and B. D. Wandelt, arXiv:0712.1148 [astro-ph].

[12] B. Chen, Y. Wang and W. Xue, arXiv:0712.2345 [hep-th].

[13] R. Brandenberger, B. Chen, and S. Koh, in preparation.

[14] B. Chen, Y. Wang, W. Xue and R. Brandenberger, arXiv:0712.2477 [hep-th].

[15] If a running of spectral index is not taken into account, the best fit values[9] of the spectral index is $n_{s}=0.958 \pm 0.016$. On the contrary, if a running spectral index is included, while $n_{s}>1$ is favored on the scale $k_{0}=0.002 \mathrm{Mpc}^{-1}, n_{s}<1$ is favored at $k_{0}=0.05 \mathrm{Mpc}^{-1}$.

[16] More exact form of the spectral index is given in Ref. [5] using $\rho \propto a^{-3(1+w)} \propto \eta^{-\frac{6(1+w)}{1+3 w}}$ at the horizon crossing $\left(k c_{s} \eta=1\right)$. In this paper, we have neglected the variations of $H$ during 
inflation period. 